\title{
BMJ Open Use of rating of perceived exertion during anterior cruciate ligament reconstruction and knee osteoarthritis rehabilitation: a scoping review protocol
}

\author{
Daniel Germano Maciel (D) , Mikhail Santos Cerqueira, \\ Wouber Hérickson de Brito Vieira
}

To cite: Germano Maciel D, Cerqueira MS, Brito Vieira WH. Use of rating of perceived exertion during anterior cruciate ligament reconstruction and knee osteoarthritis rehabilitation: a scoping review protocol. BMJ Open 2021;11:e055786. doi:10.1136/ bmjopen-2021-055786

- Prepublication history and additional supplemental material for this paper are available online. To view these files, please visit the journal online (http://dx.doi.org/10.1136/ bmjopen-2021-055786)

Received 27 July 2021 Accepted 12 November 2021

Check for updates

(C) Author(s) (or their employer(s)) 2021. Re-use permitted under CC BY-NC. No commercial re-use. See rights and permissions. Published by BMJ.

Neuromuscular Performance Laboratory, Physical Therapy Department, Federal University of Rio Grande do Norte, Natal, Rio Grande do Norte, Brazil

Correspondence to Dr Wouber Hérickson de Brito Vieira;

hericksonfisio@yahoo.com.br

\section{ABSTRACT}

Introduction Anterior cruciate ligament (ACL) tear and knee osteoarthritis (KOA) are the most prevalent musculoskeletal disorders of the knee. Rehabilitation with progressive resistance training is recommended for both disorders. Rating of perceived exertion (RPE) is widely used to prescribe, monitor, and control exercise load. However, the lack of detailed methodological description and variability in the use of RPE may hinder its validity. This scoping review summarises methodological aspects of the use of RPE in resistance exercises during ACL reconstruction and KOA rehabilitation. We also aim to identify possible methodological issues related to the use of RPE and provide recommendations for future studies. Methods and analysis This scoping review protocol was developed following the Joanna Briggs Institute and Preferred Reporting Items for Systematic Reviews and Meta-Analyses Statement extension for Scoping Reviews. The search will be conducted in Medline/PubMed, Embase, CINAHL, PEDro, Central and SPORTDiscus databases. The terms "anterior cruciate ligament rehabilitation", "knee osteoarthritis" and "resistance exercise" and their synonymous will be used isolated and combined (boolean operators AND/OR/NOT). Two reviewers will independently conduct title and abstract screening and evaluate full texts of potentially eligible articles. Data related to study design, sample, intervention characteristics and RPE outcomes will be extracted, summarised and qualitatively analysed. Ethics and dissemination The proposed scoping review does not require ethical approval since it will synthesise information from publicly available studies. Regarding dissemination activities, results will be submitted for publication in a scientific journal and presented at conferences in the field.

\section{INTRODUCTION}

Anterior cruciate ligament (ACL) tear is a common orthopaedic knee injury with annual incidence of 68.6 per 100000 personyears in the US population. ACL reconstruction (ACLR) is the treatment performed in $75 \%$ of the cases. ${ }^{1}$ Patients with ACL tear, submitted or not to reconstruction, have a relative risk of 3.84 of developing moderate
Strengths and limitations of this study

- This is a novel scoping review to understand how rating of perceived exertion (RPE) is used in anterior cruciate ligament reconstruction and knee osteoarthritis rehabilitation.

- The search strategy will involve five electronic databases including search in the grey literature.

- This study is following the international recommendation for developing a scoping review.

- The methods of this scoping review do not allow concluding whether results of included intervention studies were influenced by methodological inconsistencies related to the use of RPE.

to severe knee osteoarthritis (KOA) ${ }^{2} \mathrm{KOA}$ is a multifactorial degenerative joint condition affecting $16 \%$ of adults aged over 40 worldwide $^{3}$ and one of the leading causes of global disability. ${ }^{4}$ In both knee conditions (ACL tear and KOA), patients present quadriceps arthrogenic inhibition, ${ }^{56}$ leading to muscle atrophy, strength deficits and impaired physical function. ${ }^{78}$

Resistance exercise is the primary strategy to restore strength and functional independence following $\mathrm{ACLR}^{9}$ or $\mathrm{KOA}^{10}$ In this context, measures of muscular performance (eg, isokinetic dynamometry, 1-repetition maximum test, 10-repetition maximum test and repetitions to failure) may provide useful information for determining adequate exercise load, thus maximising muscle adaptations while minimising injury/harm risks. ${ }^{11} 12$

After appropriate measurement, load progression is required to increase muscular capacity, ${ }^{13}$ and its management should consider both external and internal load control. ${ }^{14}$ Rating of perceived exertion (RPE) is an internal loading control method widely used for prescribing and monitoring resistance exercise intensity during ACLR ${ }^{15-17}$ and 
KOA rehabilitation. ${ }^{18}{ }^{19} \mathrm{RPE}$ is defined as 'the feeling of how heavy and strenuous a physical task is ${ }^{20}$ and it can be measured using scales like Borg 6-20, Category-ratio 10 , Category ratio $100, \mathrm{OMNI}$ and repetitions in reserve.

However, in exercise science, methodological inconsistencies of the use of RPE may result in data misinterpretation and hinder measurement validity. ${ }^{21}$ Methodological inconsistencies affecting RPE may include the variety of RPE scales, RPE definitions and terms included within these definitions (eg, fatigue, heavy and discomfort) and RPE acquired from specific muscle groups, cardiorespiratory system or the whole body. ${ }^{21}$ Especially in ACLR and KOA rehabilitation, little attention is given to these inconsistencies. Several studies did not (1) describe instructions given to participants for reporting $\mathrm{RPE}^{1822-25}$; (2) specify the moment of RPE acquisition (eg, after each set, exercise or session $)^{182425}$ and (3) explain to participants whether they should report local or global RPE. ${ }^{26} 27$

Poor methodological description and lack of uniformity in the use of RPE across studies may hinder validity of RPE scales in ACLR and KOA rehabilitation and increase the likelihood of under-or overestimating of progressive resistance exercise. Moreover, to the best of our knowledge, no studies verifying the methodological aspects of RPE use in the rehabilitation of ACLR and KOA were found. Therefore, this scoping review will summarise methodological aspects of the use of RPE in resistance exercises during ACLR and KOA rehabilitation. We also aim to identify possible methodological issues related to the use of RPE and provide recommendations for future studies.

\section{METHODS AND ANALYSES}

This scoping review protocol was developed following the recommendations of the Joanna Briggs Institute ${ }^{28}$ and the Preferred Reporting Items for Systematic Reviews and Meta-Analyses Statement extension for scoping reviews. ${ }^{29}$ This study will follow the methodological framework suggested by Arksey and O'Malley, ${ }^{30}$ which includes the following stages: (1) identifying the research question; (2) identifying relevant studies; (3) selection of eligible studies; (4) charting the data and (5) collating, summarising and reporting the results. The final protocol was registered prospectively in the Open Science Framework on 2 April 2021 (osf.io/u8qxe).

\section{Stage 1: identifying the research question}

The overall research question are:

1. How is RPE used during ACLR and KOA rehabilitation?

2. Are there methodological concerns regarding the use of RPE during ACLR and KOA rehabilitation?

3. Is there methodological uniformity in the use of RPE during ACLR and KOA rehabilitation?

\section{Stage 2: identifying relevant studies: search strategy}

A broad systematic literature search will be conducted with no restrictions on language or year of publication.
Medline/PubMed, Embase, CINAHL, PEDro, Central and SPORTDiscus databases will be searched independently by two reviewers. The terms "anterior cruciate ligament rehabilitation", "knee osteoarthritis" and "resistance exercise" will be used isolated and combined. Search strategies developed for each database are described in online supplemental file 1. Forward and backward citation tracking and citation tracking of the reference lists of the included studies will be conducted, and grey literature (eg, clinical trials registers, conference proceedings and Ph.D. dissertation) will also be investigated.

\section{Stage 3: study selection}

Articles must fulfil the following inclusion criteria to be selected: (1) include participants over 18 years old who underwent ACLR (using any type of graft; eg, allograft, hamstrings or patellar tendon) or with KOA according to any diagnosis criteria (eg, American College of Rheumatology, Kellgren and Lawrence system, Osteoarthritis Research Society International), and without restrictions regarding the level of severity; (2) involve resistance training (land or aquatic; for example, using bodyweight, machines, dumbbells, elastic or floaters as external load); (3) use RPE to prescribe, monitor or adjust resistance exercise load and (4) be an intervention study, that is, randomised controlled trial, quasi-experimental study, case study or randomised controlled trial protocol. Studies will be excluded if (1) they are validation studies; (2) multimodal exercises included do not focus on lower limbs and knee muscle strength and (3) report an additional analysis in the same sample of a study already included.

First, articles found in the search strategy will be merged, and duplicates will be removed using Mendeley reference manager software (www.mendeley.com). Second, studies will be screened according to eligibility criteria. Last, the full text of potentially eligible abstracts will be carefully analysed. Two reviewers will independently perform searches, collect and analyse data of the studies. Disagreements will be resolved by discussion between both reviewers or consulting a third reviewer.

\section{Stage 4: charting the data}

Relevant data from included studies will be extracted using a customised data extraction form. If necessary, data extraction form will be improved until all reviewers agree with the final version. Reliability of data extraction form will be determined with the first five included studies. ${ }^{31}$ Two reviewers will use the form to independently extract data from $10 \%$ of the included studies and determine consistency of data extraction.

Data extraction will be undertaken independently by the same two reviewers. Disagreements will be resolved by discussion or consulting a third reviewer. Tables will be produced to show a summary of included studies, including the following data:

- Study information: author/year. 
Table 1 RPE outcomes that will be extracted from included studies

\begin{tabular}{|c|c|c|}
\hline Outcome & Description & Example \\
\hline Scale type & Which scale was used & $\begin{array}{l}\text { Borg 6-20, CR10, CR100, OMNI, repetitions ir } \\
\text { reserve scale, repetitions to failure scale }\end{array}$ \\
\hline Instructions given to participants & $\begin{array}{l}\text { Which instruction was given to participants } \\
\text { for reporting RPE }\end{array}$ & - \\
\hline Used terms & $\begin{array}{l}\text { Which terms were used in instructions given } \\
\text { to participants }\end{array}$ & Fatigue, heavy, hard or discomfort \\
\hline Purpose of using RPE & Which is the purpose of using RPE & Prescribe, monitor or adjust the load \\
\hline $\begin{array}{l}\text { Estimation and production } \\
\text { paradigms }\end{array}$ & Which paradigm was used & $\begin{array}{l}\text { Estimation-RPE is reported in response to a } \\
\text { predefined load. } \\
\text { Production - the participant self-regulated } \\
\text { exercise intensity by RPE level proposed by } \\
\text { authors }\end{array}$ \\
\hline $\begin{array}{l}\text { Exercise intensity (when } \\
\text { production paradigm was } \\
\text { applied) }\end{array}$ & Targeted internal load proposed by authors & Mild, moderate or high intensity \\
\hline Strategy for load adjustment & Which strategy was used to adjust load & $\begin{array}{l}\text { Increase or reduce external load, no of sets, } \\
\text { repetitions or exercises }\end{array}$ \\
\hline Local vs global RPE & $\begin{array}{l}\text { Participants were instructed to report RPE for } \\
\text { which part of the body }\end{array}$ & $\begin{array}{l}\text { Specific muscle groups, cardiovascular } \\
\text { system, or whole body }\end{array}$ \\
\hline Moment of RPE acquisition & $\begin{array}{l}\text { RPE was collected at which moment of the } \\
\text { session }\end{array}$ & After each set, exercise or session \\
\hline $\begin{array}{l}\text { Exercises in which RPE was } \\
\text { collected }\end{array}$ & RPE was collected during which exercises & Leg extension, leg press or squat \\
\hline
\end{tabular}

$\mathrm{CR}$, category ratio; RPE, rating of perceived effort.

- Sample: sample size; age; gender; knee condition (ACLR or KOA); time from surgery for patients with ACLR; time from beginning of symptoms for patients with KOA; level of severity (eg, Western Ontario and McMaster Universities (WOMAC), Lequesn, Lysholm, ACL-RI).

- Intervention: exercises (eg, leg press, squat and leg extension); training frequency (days per week); training duration (weeks); type of muscle contraction; external load; exercise protocol (number of series and repetitions, and resting period); strategies for load prescription, adjustment, control and progression; other interventions associated with resistance exercises (eg, neuromuscular electrical stimulation).

- RPE outcomes: scale type and purpose; instructions given to participants, including used terms; estimation and production paradigm; exercise intensity; local and global RPE; moment of RPE acquisition; exercises in which RPE was collected. Details of each item can be found in table 1 .

- Study design: randomised controlled trial, quasiexperimental study, case study and protocol for intervention study.

\section{Stage 5: collating, summarising and reporting the results}

Results regarding RPE outcomes will be summarised using a qualitative descriptive approach.
Patient and public involvement

Patients will not be involved.

\section{ETHICS AND DISSEMINATION}

This scoping review will not require ethical approval since it will synthesise information from publicly available studies. Regarding dissemination activities, results will be submitted for publication in a scientific journal and presented at conferences in the field.

Acknowledgements Authors would like to thank Coordination for the Improvement of Higher Education Personnel (CAPES, Brazil), Finance code 001, for the scholarship concession to Ingrid Martins de França, Daniel Germano Maciel and Mikhail Santos Cerqueira. Additionally, authors thank Probatus Academic Services for providing scientific language translation, revision and editing.

Contributors Conceived and designed: DGM, MSC and WHBV. Revised the paper critically: DGM, MSC and WHBV. Approved the final version: DGM, MSC and WHBV.

Funding The authors have not declared a specific grant for this research from any funding agency in the public, commercial or not-for-profit sectors.

Competing interests None declared.

Patient consent for publication Not applicable.

Provenance and peer review Not commissioned; externally peer reviewed.

Supplemental material This content has been supplied by the author(s). It has not been vetted by BMJ Publishing Group Limited (BMJ) and may not have been peer-reviewed. Any opinions or recommendations discussed are solely those of the author(s) and are not endorsed by BMJ. BMJ disclaims all liability and responsibility arising from any reliance placed on the content. Where the content includes any translated material, BMJ does not warrant the accuracy and reliability 
of the translations (including but not limited to local regulations, clinical guidelines, terminology, drug names and drug dosages), and is not responsible for any error and/or omissions arising from translation and adaptation or otherwise.

Open access This is an open access article distributed in accordance with the Creative Commons Attribution Non Commercial (CC BY-NC 4.0) license, which permits others to distribute, remix, adapt, build upon this work non-commercially, and license their derivative works on different terms, provided the original work is properly cited, appropriate credit is given, any changes made indicated, and the use is non-commercial. See: http://creativecommons.org/licenses/by-nc/4.0/.

ORCID iD

Daniel Germano Maciel http://orcid.org/0000-0003-0975-5998

\section{REFERENCES}

1 Sanders TL, Maradit Kremers H, Bryan AJ, et al. Incidence of anterior cruciate ligament tears and reconstruction: a 21-year populationbased study. Am J Sports Med 2016;44:1502-7.

2 Ajuied A, Wong F, Smith C, et al. Anterior cruciate ligament injury and radiologic progression of knee osteoarthritis: a systematic review and meta-analysis. Am J Sports Med 2014;42:2242-52.

3 Cui A, Li H, Wang D, et al. Global, regional prevalence, incidence and risk factors of knee osteoarthritis in population-based studies. EClinicalMedicine 2020;29-30:100587-13.

4 Cross M, Smith E, Hoy D, et al. The global burden of hip and knee osteoarthritis: estimates from the global burden of disease 2010 study. Ann Rheum Dis 2014;73:1323-30.

5 Sonnery-Cottet B, Saithna A, Quelard B, et al. Arthrogenic muscle inhibition after $A C L$ reconstruction: a scoping review of the efficacy of interventions. Br J Sports Med 2019;53:289-98.

6 Callaghan MJ, Parkes MJ, Hutchinson CE, et al. Factors associated with arthrogenous muscle inhibition in patellofemoral osteoarthritis. Osteoarthritis Cartilage 2014;22:742-6.

7 Schmitt LC, Paterno MV, Hewett TE. The impact of quadriceps femoris strength asymmetry on functional performance at return to sport following anterior cruciate ligament reconstruction. J Orthop Sports Phys Ther 2012;42:750-9.

8 Culvenor AG, Ruhdorfer A, Juhl C, et al. Knee extensor strength and risk of structural, symptomatic, and functional decline in knee osteoarthritis: a systematic review and meta-analysis. Arthritis Care Res 2017;69:649-58.

9 Welling W, Benjaminse A, Lemmink K, et al. Progressive strength training restores quadriceps and hamstring muscle strength within 7 months after ACL reconstruction in amateur male soccer players. Phys Ther Sport 2019;40:10-18.

10 Vincent KR, Vincent HK. Resistance exercise for knee osteoarthritis. Pm R 2012;4:S45-52.

11 Glasgow P, Phillips N, Bleakley C. Optimal loading: key variables and mechanisms. Br J Sports Med 2015;49:278-9.

12 Young JL, Rhon DI, Cleland JA, et al. The influence of exercise dosing on outcomes in patients with knee disorders: a systematic review. J Orthop Sports Phys Ther 2018;48:146-61.

13 Gabbett T, Sancho I, Dingenen B, et al. When progressing training loads, what are the considerations for healthy and injured athletes? Br J Sports Med 2021;55:947-8.
14 Scott BR, Duthie GM, Thornton HR, et al. Training monitoring for resistance exercise: theory and applications. Sports Med 2016;46:687-98.

15 Erickson LN, Lucas KCH, Davis KA, et al. Effect of blood flow restriction training on quadriceps muscle strength, morphology, physiology, and knee biomechanics before and after anterior cruciate ligament reconstruction: protocol for a randomized clinical trial. Phys Ther 2019;99:1010-9.

16 Gerber JP, Marcus RL, Dibble LE, et al. Effects of early progressive eccentric exercise on muscle structure after anterior cruciate ligament reconstruction. J Bone Joint Surg Am 2007;89:559-70.

17 Gerber JP, Marcus RL, Dibble LE, et al. Effects of early progressive eccentric exercise on muscle size and function after anterior cruciate ligament reconstruction: a 1-year follow-up study of a randomized clinical trial. Phys Ther 2009;89:51-9.

18 Vincent KR, Vasilopoulos T, Montero C, et al. Eccentric and concentric resistance exercise comparison for knee osteoarthritis. Med Sci Sports Exerc 2019;51:1977-86.

19 Primeau CA, Birmingham TB, Moyer RF, et al. Trajectories of perceived exertion and pain over a 12-week neuromuscular exercise program in patients with knee osteoarthritis. Osteoarthritis Cartilage 2020;28:1427-31.

20 Borg G. Borg's perceived exertion and pain scales. Champaign, IL: Human Kinetics, 1998: 104. vii.

21 Halperin I, Emanuel A. Rating of perceived effort: methodological concerns and future directions. Sports Med 2020;50:679-87.

22 Foroughi N, Smith RM, Lange AK, et al. Lower limb muscle strengthening does not change frontal plane moments in women with knee osteoarthritis: a randomized controlled trial. Clin Biomech 2011;26:167-74.

23 Foroughi N, Smith RM, Lange AK, et al. Progressive resistance training and dynamic alignment in osteoarthritis: a single-blind randomised controlled trial. Clin Biomech 2011;26:71-7.

24 Waller B, Munukka M, Rantalainen T, et al. Effects of high intensity resistance aquatic training on body composition and walking speed in women with mild knee osteoarthritis: a 4-month RCT with 12-month follow-up. Osteoarthritis Cartilage 2017;25:1238-46.

25 de Paula Gomes CAF, Leal-Junior ECP, Dibai-Filho AV, et al. Incorporation of photobiomodulation therapy into a therapeutic exercise program for knee osteoarthritis: a placebo-controlled, randomized, clinical trial. Lasers Surg Med 2018;50:819-28.

26 Baker KR, Nelson ME, Felson DT, et al. The efficacy of home based progressive strength training in older adults with knee osteoarthritis: a randomized controlled trial. J Rheumatol 2001;28:1655-65.

27 Vincent KR, Vincent HK. Concentric and eccentric resistance training comparison on physical function and functional pain outcomes in knee osteoarthritis: a randomized controlled trial. Am J Phys Med Rehabil 2020;99:932-40.

28 Peters MDJ, Godfrey CM, Khalil H, et al. Guidance for conducting systematic scoping reviews. Int J Evid Based Healthc 2015;13:141-6.

29 Tricco AC, Lillie E, Zarin W, et al. PRISMA extension for scoping reviews (PRISMA-ScR): checklist and explanation. Ann Intern Med 2018;169:467-73.

30 Levac D, Colquhoun H, O'Brien KK. Scoping studies: advancing the methodology. Implement Sci 2010;5:69.

31 Chen ML, Yao L, Boger J, et al. Non-Invasive brain stimulation interventions for management of chronic central neuropathic pain: a scoping review protocol. BMJ Open 2017;7:e016002. 